Est Ag 35 (2000) 271-290

\title{
Estructura de la Regla de San Agustín (IX) ${ }^{1}$
}

\section{Visión de conjunto de la Regla}

Yendo en busca de la estructura de la Regla de san Agustín, en los artículos precedentes hemos analizado varios textos agustinianos que se encuadran en diversos géneros literarios: apologético (De moribus ecclesiae catholicae 31,65-67 y De uera religione 37,68-54,106), de confesión -tanto referida al presente del autor (libro décimo de las Confessiones) como al pasado (libros 1-9 de la misma obra)-, epistolar (Epistula 48), de predicación -tanto en contexto litúrgico (Sermones sobre la Cuaresma) como exegético (Enarratio in psalmum 37)-. Concluidos ya los análisis, procede dar el paso a la síntesis y a las conclusiones.

Los análisis nos han dejado abundante material que nos permite conocer la estructura de la Regla y nos posibilita una más exacta comprensión de la misma. Pero antes cabe preguntarse si es conforme a razón utilizar con ese fin los datos que aportan los textos analizados. Partiendo de la autenticidad agustiniana del escrito ${ }^{2}$, tal extrapolación la juzgamos legítima. De una parte, dos de esos textos son en buena medida autobiográficos y compartimos la opinión de quien afirma que «no hay mejor interpretación de una Regla monástica que la vida de quien la redactó, cuando es un santo»³ y monje, añadimos. De hecho, en pocos autores aparece tan evidente como en san Agustín la afirmación, a menudo repetida, de que en espiritualidad la persona y la doctrina son

1. Cf. Estructura de la Regla de san Agustín (I-VIII), en Estudio Agustiniano 32 (1997) 407-430; 33 (1998) 5-53; 227-270; 487-524; 34 (1999) 5-29; 219-247; 539-576; 35 (2000) 49-77.

2. Si en otros tiempos fue objeto de discusión hoy está ya fuera de toda duda. Los argumentos de L. Verheijen (cf. La Règle de saint Augustin. II. Recherches historiques, París 1967) rematan los aducidos por otros estudiosos.

3. C. VACA, EL cuidado del cuerpo, en Revista Agustiniana de Espiritualidad 2 (1961) 5465; en concreto, p. 59. Remitiendo al libro décimo de las Confesiones, escribe M. Neusch: «Sur la vie monastique, le meilleur traité qu'Augustin ait écrit est sa propre vie» (La structure de la Règle, en Itinéraires augustiniens 4 [1990] 12-19; en concreto, p. 19. 
inseparables. Ya E. Gilson constataba la preocupación constante del santo por codificar los resultados de su experiencia personal4; F. van Fleteren prefiere hablar de su tendencia a universalizarlos 5 . De otra parte, dos de los siete textos examinados, De moribus ecclesiae catholicae y Epistula 48, presentan ideales específicamente monásticos; el libro décimo de las Confessiones consiste en una confesión de Agustín obispo, pero también monje6; los nueve precedentes cabe considerarlos como el relato de la vocación monástica de Agustín7; los mismos sermones sobre la Cuaresma están inspirados en el ideal monástico, que el pastor propone a imitación de sus fieles de Hipona para ese tiempo fuerte, como se indicó en su momento; ni siquiera el texto de De uera religione cabe desvincularlo de un ideal de perfección: basta tener en cuenta que Agustín presenta al cristianismo como el lugar donde toma cuerpo el ideal que los filósofos de la antigüedad sólo habían soñado sin haber conseguido darle vida ${ }^{8}$. En definitiva, sólo la Enarratio in psalmum 37, que, por otra

4. Cf. Introduction è l'étude de saint Augustin, Paris 19493, p. 31. «... le esperienze vitali... soggiacciano alle concezioni teoretiche di Agostino» (L. F. Pizzolato, introducción al libro IV, en Sant'Agostino. Confessioni. Volume II. Libri IV-VI. A cura di P. Cambronne, L. F. Pizzolato, M. Simonetti, P. Siniscalco. Traduzione di G. Chiarini, Fondazione Lorenzo Valla 1993, p. 146. Que el pensamiento del santo ha evolucionado con el tiempo es algo que él mismo reconoce (cf. Epist.143,2 CSEL 44,251; Retractationes, Prol. 1,3; 1,17 CSEL 36/2,9-10. 86-87). El paso del marco filosófico de la época inmediatamente posterior a su conversión a otro fundamentalmente bíblico vinculado a su tarea pastoral como ministro de la Iglesia implicó sin duda una evolución, pero casi siempre en la misma dirección. Una excepción es el cambio experimentado en la comprensión de la relación entre la libertad y la gracia con motivo de la consulta que le hizo el obispo de Milán Simpliciano, a la que dio respuesta en su obra Quaestiones ad Simplicianum, libri II.

5. «Augustine... has a well-known tendency to universalize his own experiencie. In the Confessiones and elsewhere, he exhibits a conversion mentality which undoubtedly arises out of his own experiencie. He is inclined to wish for others what he himself has experienced. His own conversion served as a paradigm for all mankid. For Augustine, the typical and universal value of his story in the Confessiones is beyond question» (F. Van FLETEREN, Augustine's Theory of Conversion, en Augustine: Second Founder of the Faith, Collectanea Augustiniana I, eds. J. Schnaubelt, F. v. Fleteren, New York; Peter Lang 1990, p. 65-80; en concreto, p. 67).

6. En contra L. Cilleruelo: «La misma Regla, según todas las probabilidades, fue escrita cuando san Agustín no era ya monje, sino que era un obispo preocupado de la disciplina de un monasterio por ciertos abusos que habían sobrevenido» (Diálogo fraterno, en Revista Agustiniana de Espiritualidad 4 [1963] 123-131; en concreto, p. 129).

7. Así concluye el relato de la conversión: «Conuertisti enim me ad te, ut nec uxorem quaererem nec aliquam spem saeculi huius...» (Conf. 8,12,30 CC 27,132). Y tras señalar que fue bautizado, escribe: «Simul eramus, simul habitaturi placito sancto. Quaerebamus quisnam locus nos utilius haberet seruientes tibi» (Ib. 9,8,17 CC 27,143).

8. Aunque Agustín menciona explícitamente a Platón, el ideal que propone es «pitagórico». Cf. L. Verheijen, "Vers la beauté spirituelle» (Par les praecepta uiuendi à la spiritalis pulchritudo. "Pythagore», le De Ordine de saint Augustin et sa Règle), en Augustiniana 22 (1972) 469-510 NA I,201-242; en concreto, pp. 474.480.484 NA I,206.212.216. 
parte, es el texto en que el paralelismo es más incompleto y tenue, carece de relación con un ideal específico monástico o de perfección. Por último, resulta lógica, si se acepta la organicidad y unidad global del pensamiento del obispo de Hipona, algo que nos parece debe quedar fuera de toda discusión ${ }^{9}$, aun en la variedad de formas ${ }^{10}$. Y precisamente esa unidad y organicidad explica que, a la inteligencia de un texto, en este caso su Regla monástica, sirva no sólo el examen del contexto inmediato, sino también el estudio de los principios generales que sostienen toda su espiritualidad e incluso su filosofía y teología11. Por tanto, una vez descubierta una fundamental correlación entre estructura formal y contenido doctrinal, aunque con notable riqueza termi-

\section{Cf. É. Gilson, Introduction, p. 310-311.}

10. «Malgré l'unité de l'oeuvre de saint Augustin présente dans ses profondeurs, elle se présente aux yeux sous des aspects les plus variés" (L. Verjeihen Les sermons de saint Augustin pour la carême (205-211) et sa motivation de la vie "ascetique», en Augustiniana 21 (1971) 358-404; en concreto, pp. 361-362 NA I,157-158. Se podría aplicar al tema que nos ocupa lo que un agustinólogo afirmaba sobre los comentarios del santo a la parábola del hijo pródigo: «Agustín se repite siempre, sin repetirse jamás; cada comentario y cada frase tiene su riqueza propia, su estilo y melodía peculiar, sus frases sorprendentes, llenas de encanto y de novedad» (J. ORoz RetA, Las Confesiones de san Agustín, en Augustinus 32 (1987) 133-148; en concreto, p. 145).

11. Se trata de algo que, ya tiempo atrás, reclamaban algunos autores a propósito no específicamente de la Regla, sino del monacato agustiniano en general. Así J. Morán: «... la doctrina monástica agustiniana no puede reducirse, por consiguiente, a los textos monásticos, sin sus múltiples relaciones y sin la fundamentación que los sostiene... (los autores) no han ensamblado la doctrina monacal en el cuadro de la doctrina general agustiniana. La línea que perseguimos en nuestro estudio es justamente esa: integrar la doctrina monacal en la doctrina general de san Agustín, probar que su monacato era el fruto maduro de sus concepciones filosóficas sobre lo común que habían de hallar su correspondencia en la Escritura y, por tanto, una fundamentación teológica» (El equilibrio, ideal de la vida monástica en san Agustín, Valladolid 1964, pp. 17.21). O L. Cilleruelo: «El monacato es una parte del programa general: lo reclaman la filosofía y la teología de Agustín y debe responder a las necesidades del sistema y a la salud del organismo general. De esto se deduce una conclusión: para conocer el ideal de los otros fundadores, basta conocer su Regla, sus Constituciones, Ios Reglamentos y los escritos monásticos. Empero, para conocer el monacato agustiniano, es necesario estudiar la filosofía y la teología de san Agustín, ya que este monacato es un capítulo, todo lo importante que se quiera, de una filosofía y una teología. El significado de Agustín en la historia del monacato es la misma que en la historia de la filosofía y la teología" (Caratteri del monacato agostiniano, en Sanctus Augustinus vitae spiritualis Magister, I, 43-75, Roma 1959; en concreto, pp. 43-44).

Por su parte, L. Verheijen expresa brevemente de esta manera la relación entre la Regla y las demás obras de san Agustín: «J'ai découvert qu'il est intéressant et éclairant de lire l'oeuvre, l'inmense oeuvre, d'Augustin a la lumière de la Règle et de lire la Règle à la lumière des oeuvres d'Augustin" (La Règle de saint Augustin comme prisme pour une lecture "orientée» de ses oeuvres, en Nouvelle Approche de la Règle de saint Augustin, t. II. Chemin vers la vie heureuse, Louvain 1988, pp. 60-73; en concreto, p. 61). 
nológica y variedad de criterios de organización y de perspectivas, entre diversos textos, procedentes de diferentes contextos y distintos géneros literarios, nada impide otorgar los mismos contenidos doctrinales a otro documento en el que se ha descubierto idéntica estructura formal. El documento al que ahora nos referimos es la Regla.

El presente capítulo constará de cuatro partes. Las tres primeras se ocuparán, respectivamente, de la estructura de la Regla, de su interpretación y de su dinamismo interior; en la cuarta sugerimos diversos epígrafes para cada una de sus divisiones y subdivisiones, relacionados con las distintas claves de interpretación previamente señaladas.

\subsection{Estructura de la Regla}

Comenzamos el estudio con una referencia al arte de la arquitectura. Afirmamos entonces que es uno de tantos cauces de que dispone el historiador para conocer la mentalidad de una época. De igual manera, concluíamos, la estructura de las obras literarias se constituye en rica fuente informativa sobre el contenido y mensaje de las mismas. De acuerdo con el principio formulado, teniendo siempre en mente una mejor comprensión de la Regla, en los artículos anteriores no hemos buscado otra cosa que tratar de descubrir su estructura. Nuestro esfuerzo se ha visto recompensado con resultados que juzgamos interesantes.

\subsubsection{Método seguido para detectar la estructura}

Hay edificios que tienen cada una de sus secciones interiores perfectamente divididas. Los tabiques demarcan el espacio reservado a cada sala, a la que se ha asignado una función específica. De esta manera, su estructura se hace físicamente visible a los ojos y quien entra allí la percibe de inmediato. Pero hay otro tipo de construcciones en que la división, aunque existe en la mente del arquitecto, no llega a materializarse físicamente. Cuando hizo el diseño, tuvo en cuenta los ámbitos requeridos en función de las necesidades; sin llegar a separarlos por paredes o tabiques, él tenía en mente determinado número de espacios con sus dimensiones y características propias. El visitante no los ve separados con sus ojos, pero allí tienen su lugar reservado.

Apliquemos lo dicho a una biblioteca, por ejemplo. A la hora de diseñarla, el arquitecto habrá ideado un espacio para sala de lectura, otro para despacho del bibliotecario, otro para fichero, otro para revistero, otro para depó- 
sito de revistas, otros para las distintas clases de libros: incunables, antiguos, modernos, y subdividiendo luego según las diversas ramas del saber: libros de literatura, de historia, de filosofía, teología, ciencias sociales, informática, etc. En función de los intereses de quien le confió la obra, en un caso el arquitecto puede haber separado con tabiques cada uno de los espacios indicados, $\mathrm{y}$ en otro puede haber dejado la superficie entera sin dividir físicamente. Desde el punto de vista de lo que perciben los ojos, aparecen dos modelos de bibliotecas, pero en cuanto tales, son idénticas: la una es copia de otra; la estructura es idéntica. En ambas se puede poner el mismo mobiliario, instalar idénticas estanterías y colocar los mismos libros en el mismo orden. La diferencia es que en una los ojos perciben dicha estructura y en la otra no.

Supongamos que ambas bibliotecas -entendidas como espacio físicoestán ya llenas de libros, cada uno en su sección correspondiente, conforme al plano del arquitecto. Si alguien, procedente de aquella cuyas secciones están físicamente separadas y cuya estructura ha ponderado, llega a la otra, creerá que esta carece de toda estructura; juzgará que se trata simplemente de una nave repleta de libros. Pero, si se detiene a examinar qué libros están depositados en cada espacio, percibirá la coincidencia plena con la otra y descubrirá que, más allá de lo que ven los ojos, la estructura ideal de una y otra es idéntica. No hace falta que haya demarcaciones físicas. La homogeneidad del material almacenado en cada espacio establece de hecho demarcaciones $y, a$ partir de él, se puede llegar a percibir la estructura subyacente.

Este último caso es el que se da en la Regla. Es sabido que la división en capítulos no procede de su autor. El texto fue escrito de corrido, sin las divisiones a que nos han acostumbrado los editores modernos. El arquitecto Agustín prefirió dejar la Regla, en la que introdujo sus prescripciones monásticas, a modo de nave industrial sin pared o tabique físico alguno. Pero eso no quiere decir que en su mente no tuviera una estructura neta y definida, a que se ajustan las prescripciones mencionadas. Ahora bien, ¿cómo descubrirla? Utilizando la imagen anterior, hemos visitado y examinado detenidamente otras «bibliotecas» diseñadas por el mismo san Agustín, en las que la estructura se manifestaba más formal, clara o explícita. Hemos tomado nota de dicha estructura, de las divisiones y subdivisiones de sus espacios, e igualmente del material almacenado en cada uno de ellos. Después hemos vuelto a la Regla y con no menor atención nos hemos fijado en sus contenidos cual material almacenado en ella. Una comparación ulterior nos ha permitido descubrir en ella la misma estructura que sostenía las otras construcciones del arquitecto san Agustín sometidas a examen. Pudimos ver cuán fácil era introducir los contenidos de la Regla en los distintos espacios de aquella. Ello tenía fácil explicación: la estructura era la misma. Por las razones que fueran, 
Agustín había renunciado a separar las salas de la Regla, pero el espacio para cada una de ellas allí estaba.

\subsubsection{La estructura detectada}

Siguiendo con la imagen arquitectónica, podemos hablar, a propósito de la Regla, de plano de conjunto y planos de detalle. L. Verheijen supo ver bien el primero, pero no se percató de los segundos. Acertadamente advirtió que la edificación constaba de tres cuerpos ${ }^{12}$ (que hemos denominado secciones A, B y C), pero le faltó acercarse un poco más. Al examinar cada uno de esos cuerpos más de cerca, hemos podido descubrir que el primero está constituido por un único y amplio espacio; que el segundo consta de tres compartimientos -unidades en nuestra terminología-, con ulteriores divisiones -llamémoslas salas- en cantidad idéntica al número de serie: una sola en el compartimiento primero, dos en el segundo y tres en el tercero; que el tercero tiene una distribución especial: en el nivel «vertical» consta de dos partes, la segunda de las cuales, a su vez, se subdivide en otras tres, equivalentes a las tres unidades del cuerpo segundo, de las que son correlatos.

Este sería, pues, el plano de conjunto de la Regla:

Ver esquema de la página siguiente:

Tales son las diversas estancias del edificio de la Regla. Para qué está reservada cada una de ellas irá apareciendo en las páginas que siguen y, sobre todo, en apartado posterior, al hablar de su interpretación ${ }^{13}$.

12. Además del «prólogo» y «epílogo».

13. En el esquema que ofrecimos en el primer artículo de la serie aparece ya, de forma genérica, alguna información, pero no toda la que ahora podemos ofrecer y de que entonces carecíamos. Pasa lo mismo que con el plano: el allí diseñado responde a la verdad, pero es demasiado elemental respecto del que aparece aquí. 


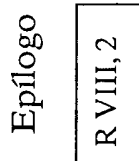
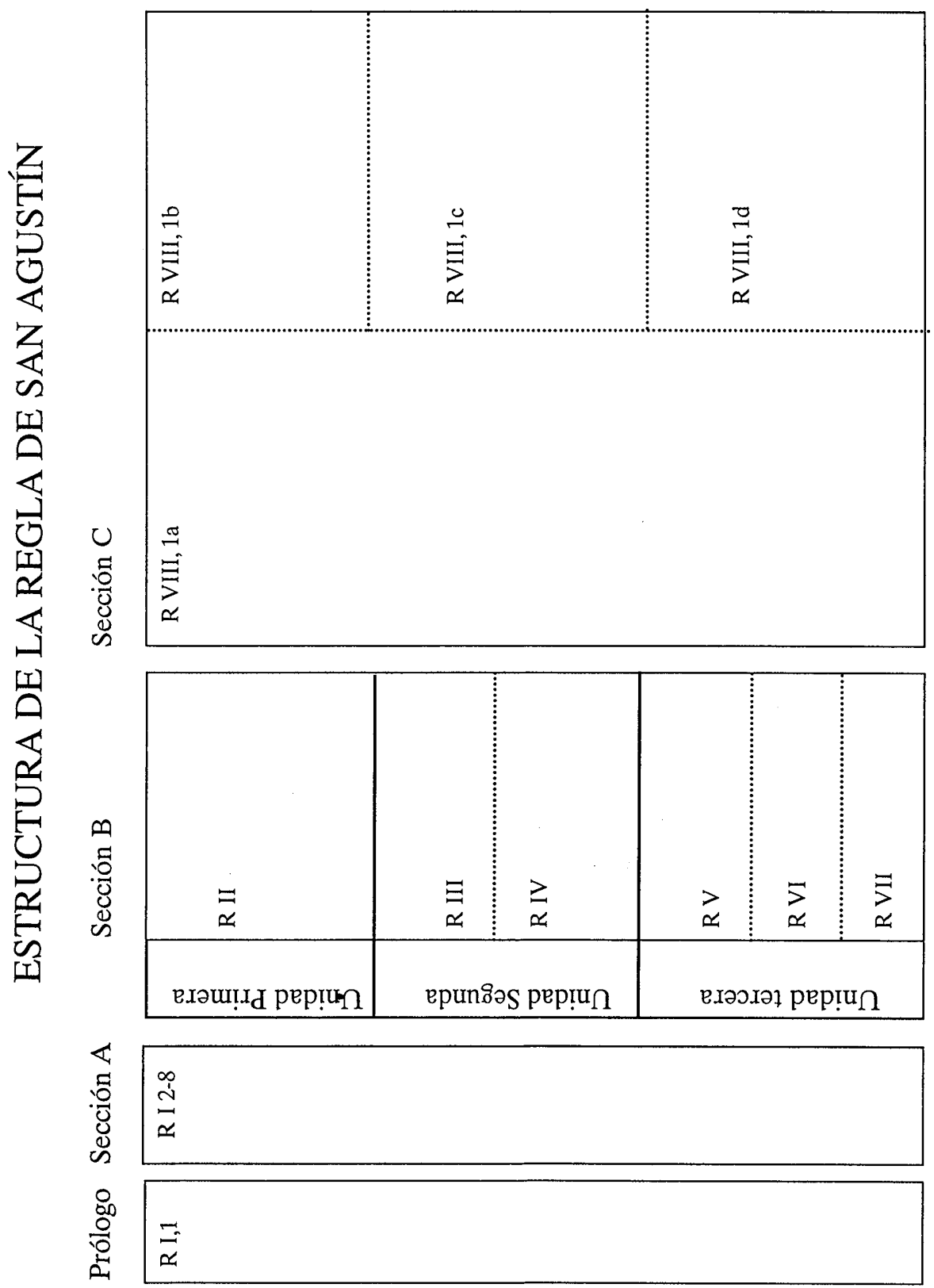


\subsubsection{Justificación de dicha estructura}

Dejamos la imagen y volvemos a nuestra terminología habitual de «secciones» $\mathrm{y}$ «unidades», que corresponden, respectivamente, al plano horizontal y al vertical o perspectiva diacrónica y sincrónica de la Regla.

\subsubsection{Justificación de la estructura horizontal14}

Como se indicó, las tres secciones ya las había señalado L. Verheijen. Más aún, habiéndolas detectado en otros textos examinados por él, había concluido que constituían una estructura de composición utilizada habitualmente por el santo ${ }^{15}$. Ninguno de los textos analizados por nosotros en los artículos precedentes está incluido dentro de los estudiados por él, pero, en su materialidad, las conclusiones a que hemos llegado no difieren en lo sustancial, esto es, en cuanto que todos ofrecen la posibilidad de hablar de tres secciones ${ }^{16}$.

El texto de mor. no ofrece una estructura totalmente lineal, pero sí un material que se encuadra sin dificultad en tres secciones: referencias ascéticas, objetivo y posibilidad de consecución. Las tres secciones aparecen, sin embargo, netas en el libro décimo de conf. como tres momentos del caminar hacia Dios, formalmente reconocidos por el obispo de Hipona. Recogen respectivamente una inicial experiencia gratificante de Dios que el santo desea hacer plena (sección A), las tres concupiscencias como impedimento que es preciso superar para conseguirlo (sección B) y la acción de Cristo mediador que lo posibilita (sección C). Asimismo en los nueve primeros libros de la misma obra tampoco ha sido difícil percibir el triple momento, con ropaje ligeramente diverso: Agustín reconoce tener en Dios, como todo hombre, el lugar de su felicidad (sección A), pero, de hecho, la buscó durante mucho tiempo en

14. Dejamos de lado el prólogo y el epílogo.

15. Cf. Estructura (I), en Estudio Agustiniano 32 (1997) 413-414, nota 26.

16. A partir de ahora las referencias a los artículos anteriores serán frecuentes. Salvo en casos contados, no las señalaremos. Nos limitamos a recordar en qué artículos -publicados siempre en Estudio Agustiniano- han sido analizados los respectivos textos: De uera religione en Estructura (II) (3 [1998] 5-53); Confessiones 10 en Estructura (III) (33 [1998] 227-270); Confessiones 1-9 en Estructura (IV) (33 [1998] 487-524); Epistula 48 en Estructura (V) (34 [1999] 5-29); Sermones sobre la Cuaresma en Estructura (VI) (34 [1999] 219-247); De moribus Ecclesiae catholicae 30,67 en Estructura (VII) (34 [1999] 539-576); Enarratio in psalmum 37 en Estructura (VIII) (35 [2000] 49-77). 
la satisfacción de las diversas apetencias terrenas (sección B), hasta que finalmente descubrió en Cristo el camino que lleva a dicha felicidad (sección C).

También ep. 48 nos brinda ese triple estadio: el objetivo por cuya consecución se esfuerza el monje (sección A), la «actividad» que lleva asociada (sección B) y las condiciones que la hacen posible (sección C). Aunque de forma menos nítida, comprensible por tratarse no de un texto único, sino de varios sermones, el mismo resultado se obtiene del análisis de los sermones sobre la Cuaresma. El objetivo último del cristiano es arribar a la pascua definitiva (sección A), simbolizada ya en el tiempo pascual, con miras a la cual se asumen las prácticas ascéticas (sección B), tomando a Jesucristo como punto de referencia (sección C).

Hemos dejado para el final el uera rel. y en. Ps. 37, primer y último texto examinados, porque ambos se separan de los demás en que, en vez de tres, presentan cuatro situaciones: dos en este mundo y otras dos en el futuro. Mientras todos los otros textos contemplan, respecto del futuro, sólo la situación de felicidad (sección A), estos contemplan también la posibilidad del fracaso o condenación eterna. Vera rel. expone cuál será la suerte futura tanto de quienes en esta vida han combatido las concupiscencias (situación $\mathrm{C}$, equivalente a la sección A de la Regla), como la de quienes las han secundado (situación $\mathrm{D}^{17}$ ) y, de modo análogo, en. Ps. 37 habla tanto del sábado futuro como de la posibilidad del castigo eterno. El carácter descriptivo del primer texto y el sometimiento al salmo que comenta en el segundo dan razón, respectivamente, de esta novedad 18 .

Leída la Regla a la luz de estos textos, se nos antoja clara su estructura tripartita. En efecto, es fácil advertir en ella una sección $\mathrm{A}$, correspondiente a su capítulo primero, que contiene el objetivo que mueve a los siervos de Dios (alcanzar a Dios: in deum $)^{19}$; una sección $\mathrm{B}$, correspondiente a los capítulos del dos al séptimo, que contiene un amplio abanico de exigencias; por último, una sección $\mathrm{C}$, correspondiente al primer parágrafo de su capítulo octavo, que señala cómo es posible satisfacerlas. Lo único que no contempla es la posibi-

17. Sin equivalente sin la Regla y otros textos analizados a excepción, en todo caso, de en. Ps. 37.

18. En cuanto a uera rel. hay que tener en cuenta también su destinatario: el mecenas de Agustín, Romaniano, al que el santo quería hacer retornar a la fe católica desde el maniqueísmo al que él mismo le había conducido. Resulta lógico que en el contexto de esa exhortación le presentase la posibilidad del fracaso definitivo si no seguía la verdadera religión.

19. Además del objetivo, la sección incluye también otros aspectos que se especificarán más adelante. 
lidad del fracaso. Cosa por otra parte comprensible: presentar esa posibilidad no es el mejor medio para lograr adeptos para la causa.

Pero a esta estructura hay que buscarle un sentido. ¿Sobre qué criterio o criterios se ha levantado? San Agustín no lo explicita en ninguno de los textos examinados. Tratar de adivinarlos, es tarea que dejó para nosotros. Moviéndose dentro de criterios materiales, L. Verheijen hablaba de parte fundamental (cuerpo primero), diversas regulaciones prácticas (cuerpo segundo) y mirada profunda y englobante a todo lo anterior (cuerpo tercero). Nosotros, tomando un criterio existencial, preferimos hablar de ideal (cuerpo primero), obstáculos (cuerpo segundo) y modo cómo superarlos (cuerpo tercero). O, con otra terminología, meta a alcanzar (cuerpo primero), situación de partida que es preciso dejar atrás (cuerpo segundo) y posibilidades de hacerlo (cuerpo tercero $)^{20}$. U, optando por un criterio más formal y filosófico, procedería hablar de unidad absoluta (cuerpo primero), multiplicidad lamentable (cuerpo segundo) y unidad relativa (cuerpo tercero). La realidad es la misma, pero contemplada desde ángulos diversos. En efecto, la meta a conseguir se identifica con la unidad absoluta; a su vez, la multiplicidad lamentable designa perfectamente la situación que es preciso superar y la unidad relativa identifica el camino a recorrer ${ }^{21}$.

\subsubsection{Justificación de la estructura vertical}

Descubiertas las tres secciones del plano horizontal, no podemos pasarlas por alto al ocuparnos de la estructura vertical. Examinaremos, por tanto, la estructura de cada una de las secciones. Consideramos las unidades según el orden siguiente: B, C y A; esto es, de mayor a menor nivel de complejidad.

20. En Estructura (II) proponíamos todavía otra: posesión plena en el futuro, privación en el presente; posesión parcial en el presente, respectivamente (cf. Estudio Agustiniano 33 [1998] p. 50).

21. Unidad y multiplicidad que quedan reflejadas en las divisiones internas de cada sección. Si hablamos de unidad, aunque relativa, a propósito de la sección $\mathrm{C}$, es debida a que los tres compartimientos se refieren a tres aspectos del único Cristo. Sobre ello hablaremos en su momento. 


\subsection{Sección B}

En la imagen, el segundo cuerpo del edificio de la Regla consta de tres compartimientos, con ulteriores divisiones en el segundo y en el tercero: dos en aquel y tres en este. Según la terminología que venimos utilizando, la sección $\mathrm{B}$ consta de tres unidades, de las cuales la segunda contiene, a su vez, dos sub-unidades y la tercera tres.

Los textos examinados nos han permitido ver que el servirse de esta específica estructura ternaria, con las modalidades señaladas, es proceder frecuente de san Agustín. En efecto, en mor. el santo considera: a) la renuncia a la satisfacción de los sentidos, en el doble ámbito, alimentario y sexual; b) el cultivo del espíritu, mediante «oraciones», lecturas y debates; c) las relaciones sociales, con tres manifestaciones concretas: sin orgullo/humildes, sin terquedad/sosegados, sin envidia/austeros. En uera rel. se ocupa de los tres vicios siguientes: a) el del placer (voluptas) con sus manifestaciones en el triple ámbito de la alimentación, del descanso y de la sexualidad; b) el de la curiosidad (curiositas) que se satisface con los espectáculos sensibles, tanto los percibidos por los sentidos como los creados por la imaginación; c) el del orgullo (superbia), puesto en relación con los vicios de la envidia y de la ira. En conf. 10 Agustín se examina acerca de: a) la concupiscencia de la carne, pasando revista a cada uno de los cinco sentidos; b) la de los ojos, centrándose en lo que personalmente le afectaba, en concreto lo que impedía o turbaba su oración; y c) la ambición mundana con sus manifestaciones: el afán de dominio sobre los demás, la búsqueda de la gloria que tributan los demás, la negativa a compartir con los demás los dones recibidos de Dios. Lo mismo se detecta en conf. 1-9, aunque él no sea explícito y cambie en cierta medida el esquema: a) respecto de la concupiscencia de la carne, considera el ámbito alimentario y el sexual; b) a propósito de la concupiscencia de los ojos hace el planteamiento desde los conceptos de interioridad y exterioridad, asociando siempre de un modo u otro la Escritura; c) en cuanto a la ambición mundana, saca a relucir el afán de dominio y honores, el deseo de riquezas, y el ansia de celebridad. En la ep. 48 presenta a los monjes ocupados en una triple actividad, a saber, a) la oración, de la que contempla las dos posibilidades: la pública y la privada; b) el ayuno/abstinencia en el ámbito alimentario y sexual; y c) la limosna en su doble modalidad: la material y la espiritual del perdón. Los sermones sobre la Cuaresma nos sitúan en el mismo escenario, con ligeras variaciones: respecto de la oración no aparece explícita la distinción entre la pública y la privada y, respecto de la limosna, hay elementos para hablar de una tercera clase a añadir a las dos ya mencionadas: la limosna del servicio. Para concluir, en en. Ps. 37, al hilo del texto del salmo, se detiene a) en las dolencias físi- 
cas (hambre, sed, fatiga), b) en las frustraciones espirituales (las «ilusiones») y c) en las injusticias sociales, en concreto en el ámbito de las riquezas y de la fama.

Estos datos nos dejan constancia de un proceder que es habitual en san Agustín. Podemos hablar de una especie de molde de que se sirve cuando se ocupa de las dimensiones ascéticas requeridas para alcanzar la perfección.

Leída la sección B de la Regla, también ella con una evidente dimensión ascética, no nos queda duda de su estructura tripartita también aquí, esto es, en el plano vertical que considera las unidades. Efectivamente, no es difícil advertir en ella una unidad primera, correspondiente a su capítulo segundo, que se ocupa de la oración (como mor., ep. 48, sermones sobre la Cuaresma), cuyos preceptos concretos, además, se integran naturalmente en el marco de la concupiscencia de los ojos (ya sea con referencia a la curiosidad, exterior o interior, como en uera rel. y conf. 10, ya sea en la dialéctica entre interioridad y exterioridad, como en conf.1-9), ya sea dentro de las enfermedades del alma (como en en. Ps. 37), ya en la relación con la Escritura (como en mor., uera rel., conf. 1-9). Una unidad segunda, correspondiente a los capítulos tercero y cuarto, que regula ascéticamente el ámbito alimentario y sexual (como en mor., conf. 1-9 y sermones sobre la Cuaresma), el ámbito alimentario, de descanso y sexual (como uera rel.), el ámbito alimentario, sexual y el de las tribulaciones físicas (como ep. 48), los cinco sentidos (como conf. 10), o busca remedio a las dolencias físicas del hambre, sed y fatiga (como en. Ps. 37). Y también una unidad tercera, correspondiente a los capítulos quinto, sexto y séptimo, donde Agustín manifiesta su deseo de que los siervos de Dios carezcan de envidia, rencor y orgullo o, positivamente, sean austeros, sosegados y humildes (como en mor.), libres del vicio de la envidia y la ira (como en uera rel.), sin autosuficiencia insolidaria, sin sucumbir a la búsqueda de la celebridad o alabanza y sin afán de dominar (como en conf. 10 ó 1-9); dispuestos a compartir y a perdonar (como en ep. 48), o a compartir, perdonar y servir (como en los sermones sobre la Cuaresma).

La lectura de la sección B de la Regla desde los textos paralelos señalados nos hace advertir algún otro aspecto. Respecto de la primera unidad, el mor. ofrecía tres elementos: oraciones, lecturas y debates. De ellos, la Regla sólo ha conservado con claridad el primero, el segundo lo deja apenas insinuado 22 , mientras que el tercero lo ha perdido del todo. Pero el documento

22. En la Regla $(I I, 4)$ hay una referencia a la Escritura. La lectura en el contexto de la unidad segunda aparece clara en C1-9 y también en uera rel. 
monástico no representa ninguna novedad al respecto. El hecho puede reflejar un aspecto de la evolución de Agustín ${ }^{23}$. Respecto de la segunda unidad, la Regla presenta a toda luz lo referente a los ámbitos alimentario y sexual, presentes en todos los demás textos, a excepción quizá de en. Ps. 37 en que el segundo queda muy diluido; además, dentro de ellos integra los diversos sentidos, seguidos como criterio en conf. 1024; no se olvida tampoco de la situación de debilidad o enfermedad mencionada en en. Ps. 37 y ep. 48; por último, aunque con una simple referencia introduce también el ámbito del descanso/fatiga, presentes en uera rel. y en. Ps. 37. En cuanto a la tercera unidad, la Regla se caracteriza por abarcar todos los elementos presentes en los otros textos, que no se presentan uniformes. En efecto, mientras unos ofrecen sólo un elemento (así conf. 9,125), otros ofrecen dos (así mor., uera rel., ep. 48 y en. Ps. 37) y otros al menos tres (es el caso de conf. 1-8 y los sermones sobre la Cuaresma). A juzgar por cuanto dicho, la Regla se nos presenta como un texto de síntesis de cuanto el santo ha ido ofreciendo en el conjunto de los otros. Es un texto integrador; de hecho sólo deja fuera los debates, en la primera unidad.

Este aspecto integrador resulta también de otra consideración. Nos referimos a los criterios organizadores de las tres unidades. Agustín no ha utilizado siempre los mismos. Estos varían según los diversos textos y en función de distintos momentos, circunstancias e intereses. Esos criterios han sido las virtudes cardinales (prudencia, templanza, fortaleza/justicia), en mor.; las tres vicios raíz (cupiditates) en uera rel. y conf. 1-9; las tres concupiscencias señaladas por san Juan (1 Jn 2,16), en conf. 10; las tres prácticas ascéticas mencionadas por Jesús en el Sermón de la montaña, en ep. 48 y en los sermones sobre la Cuaresma; por último, los males del hombre en función de su triple origen: cuerpo, alma, el prójimo, en en. Ps. 3726 . Si prescindimos de este último, se

23. Cf. L. VERHEIJEN, «Vers la beauté spirituelle» (Par les praecepta uiuendi à la spiritalis pulchritudo. «Pythagore», le De Ordine de saint Augustin et sa Règle), en Augustiniana XXII (1972) p. 501 NA I,233.

24. A excepción del sentido del olfato, pero sobre ello cf. Estructura (III), en Estudio Agustiniano 33 (1998) 235-238.246-248.

25. Debido a su carácter de síntesis de todo lo anterior; pero en conf. 4-5 ha ofrecido los elementos presentes en otros textos.

26. Al respecto puede ser ilustrativa también la referencia a otros textos agustinianos, el primero de ellos el libro décimo tercero de las Confessiones. Dentro de la interpretación alegórica que hace el santo de los días de la creación, en conexión con el día sexto -visto como momento de la segunda creación- se dirige a los espirituales con estas palabras: «No os conforméis a este siglo (1 Jn 2,16), precaveos ante él. El alma vive evitando aquellas cosas por cuyo deseo muere. Absteneos de la inhumana ferocidad del orgullo (continete uos ab inmani feritate superbiae), de la voluptuosidad indolente de la lujuria (ab inerti uoluptate luxuriae) y del 
puede ver la evolución de Agustín: el primer criterio está tomado de la filosofía del tiempo; el segundo es probablemente una elaboración personal, resultado de la fusión de filosofía y revelación; el tercero y cuarto son ya plenamente revelado, evangélico. El quinto es también teológico en el planteamiento que de él hace san Agustín. En este sentido, la lectura de la Regla nos permite advertir cómo san Agustín ha sabido elaborar un texto en que tienen cabida todos esos criterios. Esto es, se puede leer desde cualquiera de ellos.

\subsection{Sección $C$.}

La sección C de la Regla se corresponde con el primer parágrafo del capítulo octavo. En la imagen del edificio, la presentamos con dos partes, la segunda de las cuales subdividida en otras tres, denominadas por nosotros «unidades». Veamos la justificación de esta estructura, a partir de textos paralelos.

Los textos examinados no se han mostrado tan uniformes respecto de sección $\mathrm{C}$ como respecto de la $\mathrm{B}$. Se pueden establecer tres modelos. El primero señala la parte que compete a Dios en la vida ascética de los cristianos, siervos de Dios o no, según los casos. Este modelo se encuentra únicamente en mor. Allí indica el santo que los siervos de Dios han hecho lo que han hecho gracias a Él.

El segundo tiene como punto de referencia el otro polo: no ya Dios, sino los cristianos mismos. En él hay que colocar a uera rel. El texto hace saber cómo quien no se deja arrastrar por las concupiscencias, ya en esta vida y en la medida de que es capaz, a) experimentará la belleza de la Verdad y la Sabiduría, b) tendrá una sensación placentera del Señor y c) disfrutará de una

nombre engañoso de ciencia (a fallaci nomine scientiae), de modo que se amansen la fieras, queden domadas las bestias y las serpientes se vuelvan inofensivas. Se trata de sentimientos del alma expresados en forma alegórica. Por su parte, el boato del orgullo, el placer de la sensualidad y el veneno de la curiosidad son sentimientos del alma muerta» (Conf. 13,19,30 CC $27,259)$. Carece de relieve que, en este texto concreto, bajo espirituales entienda a los ministros de la iglesia que han de ser ejemplo de los fieles (antes, por el contrario, ha dicho que espirituales pueden ser tanto los que presiden como los que les obedecen [cf. qui praesunt...]); lo importante es que vincula, en otros términos, el contenido del texto de $1 \mathrm{Jn} 2,16$ con los espirituales, que los siervos de Dios ya son o, en todo caso, aspiran a ser. A su vez, del pecado de Adán surgió la salazón del mar que se extendió por doquier, esto es, el género humano con sus abismos de curiosidad, sus tormentas de orgullo y sus fluctuaciones de inestabilidad (profunde curiosum et procellose tumidum et instabiliter fluidum) (Ib.13,20,28 CC 27,257). Cf́t también las tres formas de atacar que tiene el enemigo, presentadas en el Sermo 274 PL 38,1253: por el placer, por el abuso de los poderosos, por el error. En las tres fue derrotado por el mártir, personificación aquí del cristiano espiritual. 
libertad relativa para obrar. Algo semejante advertimos en conf.1-9, donde se nos presenta Agustín que, habiéndose liberado ya de la esclavitud a las concupiscencias, ha hallado a Cristo, a) más dulce que todo placer, b) más refulgente que toda luz y c) más excelso que todo honor. En este mismo grupo cabría encuadrar tanto los sermones sobre la Cuaresma como en. Ps 37. En el primer texto, el pastor, de un lado, presenta ante los fieles el ejemplo de Cristo que a) oró, b) ayunó y c) dio limosna, como estímulo para cumplir con las prácticas ascéticas; de otro, describe a los mismos fieles ardiendo en deseos de la belleza de Jesucristo. En el segundo, al cristiano que sufre el dolor, el engaño y la injusticia se le pone ante los ojos a) el buen olor de Cristo resucitado, b) a Cristo, Verdad encarnada, y c) la paciencia de Cristo.

El tercer modelo presenta unidas las referencias que aparecieron por separado en los dos anteriores. Entre ellos hay que colocar la ep. 48 que, de una parte, presenta a Dios obrando todo en todos, en concreto en los monjes, $\mathrm{y}$, a la vez, a los monjes ardiendo gracias al Espíritu. Texto del que es preciso poner de relieve dos aspectos. El primero, que sustituye -caso único- el elemento cristológico, presente en los demás textos, por el pneumatológico. El segundo, la constatación de que no especifica, esto es, no establece relaciones diferenciadas con cada una de las unidades de la sección B. Aunque no resulte tan claro, cabe introducir aquí también a conf.10. De una parte, presenta la bondad del Padre que no perdonó a su propio Hijo sino que lo entregó por los hombres; de otra, alimenta su esperanza considerando que si grandes son sus heridas, más grande es la Medicina de que dispone: Jesucristo. Aunque aquí no especifica más, a partir del contexto se puede pensar bien en una triple relación con Él, en función de las tres clases de heridas (las tres concupiscencias).

Es difícil aunar todos estos textos en una estructura común. La más cercana a la realidad sería esta: a) + b) que incluye i), ii) y iii). En a) el protagonista es Dios que actúa de un modo u otro en favor del asceta. En b) el protagonista es el asceta con una triple mirada hacia Cristo: i) Cristo Belleza/Verdad, Sabiduría, refulgente, orante; ii) Cristo suave, dulce, bienoliente, ayunante, bello; iii) Cristo liberador, excelso, «limosnero», paciente ${ }^{27}$.

Si ahora volvemos los ojos a la sección $\mathrm{C}$ de la Regla no resulta difícil integrar su contenido en esta estructura. El texto tiene dos referencias: una a Dios, y otra a los siervos de Dios, respecto de los cuales contempla tres posibilidades, que nos dan pie para seguir hablando de tres unidades. Ya en con-

27. El elemento cristológico cede el puesto al pneumatológico en Ep. 48. 
creto, en su primer párrafo nos presenta la parte activa de Dios: «Que él os conceda cumplir todo esto» (= a: R VIII,1a). En lo que sigue, lo que compete a los siervos de Dios (= b: R VIII,1b-d); estos, a su vez, colocados en una triple relación con Jesucristo: enamorados de Él en cuanto Belleza espiritual (i: R VIII,1b); ardiendo por Él en cuanto bienoliente (ii: R VIII,1c); liberados por Él (iii: R VIII,1d). La presente estructura integra en cuadro orgánico los datos de todos los demás textos, a excepción del elemento pneumatológico, propio de la ep. 48. Al mismo tiempo, como es de suponer desde el paralelismo, todos los demás textos nos dan una clave para interpretar el correlato en la Regla.

De nuevo, también en esta sección la Regla se nos presenta como síntesis de cuantos aspectos han ido apareciendo en los diversos textos con los que la hemos comparado.

Las subdivisiones de las unidades que existían en la sección B han desaparecido. Aún se mantienen las tres, pero con un único punto de referencia: Cristo. Es un paso adelante hacia la unidad. Cristo integra en sí cuanto ofrecen las tres concupiscencias. De ahí que a este nivel, donde se le coloca como alternativa a ellas, aún aparecen las tres unidades.

\subsection{Sección A.}

La sección se corresponde con el capítulo primero de la Regla. Desde la imagen utilizada, en su momento la presentamos como un único y amplio espacio, carente de divisiones ulteriores. Su contenido lo hemos venido presentando resumido en estos puntos: el objetivo a conseguir, la modalidad de consecución, el obstáculo del orgullo, el sentido del mismo. Aunque se trata de cuatro elementos, no por ello abrimos otros tantos compartimientos dentro de este cuerpo del edificio. La razón es que no se trata de elementos paralelos o alternativos, sino que los tres restantes están al servicio del primero. Cosa distinta sería si se pudiese hablar de varios objetivos o varias modalidades, etc.

Veamos ahora qué ayuda nos aportan los textos paralelos. Aunque también aquí estamos lejos de la uniformidad absoluta, el nivel de acuerdo es considerable. Comenzamos por los dos textos específicamente monásticos: mor. y ep. 48. El primero, con la concisión que le es propia, señala el objetivo: la vida de los monjes estaba totalmente orientada hacia Dios, la modalidad comunitaria: vida común y concordia en grado superlativo, y el sentido cultual: la vida en unidad y concordia es vista como un acto de culto, una ofrenda, a Dios. El segundo habla explícitamente de un propositum monástico, que 
no es otro que re-volar a Dios; pone énfasis en que ese propositum lo han de realizar los monjes con sentido de comunión y servicio eclesial, modalidad que tiene su máximo obstáculo en el orgullo o la desidia, según las personas.

Ni conf. 10 ni conf. 1-9 son tan claros y explícitos, pero prestan, no obstante, su ayuda. En el primer texto san Agustín presenta su anhelo y objetivo: alcanzar la paz en Dios; pero recalca que quiere hacer la peregrinación hacia su consecución en compañía de los hermanos en la fe, presentada como un acto de culto. En el segundo, el santo deja bien claro cuál es el objetivo del hombre: llegar a Dios, único lugar donde hallará el descanso pleno. Pero tal descanso no lo concibe como meta individual, sino colectiva: no será sólo «su» descanso, sino también el «nuestro». Deja asimismo bien claro el obstáculo que significa el pecado y específicamente el orgullo con vistas a esa consecución. Esa conciencia de camino hacia la patria celeste es también uno de los puntos fuertes de los sermones sobre la Cuaresma, recordando asimismo que los peregrinos han de tener una única alma.

Por último, los textos de uera rel. y en. Ps 37. Ambos tienen en común el contemplar una cuarta situación, correlato negativo a la tercera (equivalente a la sección A de la Regla). Si esta señala el triunfo, aquella el fracaso. Pero aquí se acaban las coincidencias. El primero de los textos es el único que divide esta sección en tres unidades, al señalar que el hombre que no se deje arrastrar por las tres concupiscencias alcanzará en la otra vida lo que torcidamente buscaba conseguir a través de ellas: el conocimiento pleno, la salud plena y la paz perfecta. Es también el único texto en que está ausente el aspecto comunitario ${ }^{28}$. El segundo texto tiene como peculiaridad propia el designar el objetivo que se pretende conseguir como «el sábado». Comprensible esta designación porque se la ofrece el salmo que está comentando. Aunque no haga referencia a ese caminar en común, el sentido comunitario aparece claro, aunque implícito, en la doctrina del Christus totus que domina toda la enarratio.

Mirando ahora a la Regla, de nuevo encontramos en su sección A la síntesis de prácticamente todos los elementos recogidos de los textos paralelos, aunque su designación, condicionada por el contexto, sea diferente. El «prácticamente todos» tiene, está claro, un sentido restrictivo. En efecto, la Regla carece de una nueva sección que señalase el fracaso posible, presente en vera rel. y en en. ps. 37. Rompe también con uera rel. al no otorgar una estructura tripartita para el objetivo. Aunque desde un planteamiento filosófico, como

28. Es cierto que, de hecho, nadie camina sólo y que encuentra a los demás ya como apoyos, ya como estorbos; pero en el texto no aparece énfasis alguno en el caminar en común. 
era el de uera rel, tenía su lógica, para el Agustín de la Regla ha perdido su sentido: prefiere hablar no ya de los valores buscados sino del detentor de los mismos que es uno sólo: Dios. Dios resume en sí todos los valores a los que el hombre puede aspirar. Además, actuando como actuó queda en relieve el proceso de unificación que subyace en la Regla. En el plano de la misma, la sección $\mathrm{B}$ contiene seis espacios diferentes, la $\mathrm{C}$ sólo tres ${ }^{29}$, la $\mathrm{A}$, que representa la meta, ya sólo uno. Dios será todo en todos. Tampoco tiene lugar para la desidia mencionada en $e p .48$, pero se comprende por tratarse de una actitud muy concreta, que responde a una situación particular.

A la luz de los paralelos, creemos se puede mantener con suficiente fundamento la estructura de esa sección A. Dentro de la coincidencia global, el texto al que más cercano se siente la Regla es el mor.; sólo le separa de él la introducción del elemento perturbador que es el orgullo, que Agustín no introduce allí, quizá por el carácter de panegírico del texto.

\subsubsection{Juicios y valoraciones}

El lector ya conoce nuestra respuesta a la pregunta de si la Regla de san Agustín tiene estructura definida. Respuesta a todas luces positiva, amparada en la comparación con numerosos otros textos del santo, además de pedirla la lógica. Es difícil pensar, en efecto, que quien fue brillante alumno y brillante profesor de Retórica que, entre otras cosas, se ocupaba del arte de la composición, no hubiese puesto personalmente en práctica lo que había aprendido y enseñado. Procede ahora, pues, volver los ojos a las respuestas que otros estudiosos han dado a la misma pregunta, para valorarlas desde la meta alcanzada.

Hace ya más de tres décadas, el gran agustinólogo, T. van Bavel, afirmaba que san Agustín no había trabajado jamás bajo las directrices de un esquema, porque ello no iba con su carácter. Y añadía que tampoco lo había hecho en la Regla ${ }^{30}$. Afirmación difícil de sostener tanto en su alcance general como en su aplicación al caso concreto de la Regla a la luz de nuestro estudio ${ }^{31}$. El nítido plano de la Regla anteriormente diseñado contradice también la opinión

29. En la parte que toca al hombre.

30. T. van BAVEL, La espiritualidad de la Regla de san Agustín, en Augustinus 12 (1967) 433-448; en concreto, p. 438.

31. Tal planteamiento resultaba ya contradicho por el mismo L. Verheijen, que veía en la Regla un esquema de composición usado por el santo en varios otros de sus textos. Cf. Estructura (I), en Estudio Agustiniano 32 (1997) p. 414, nota 26. 
de D. Sanchis de que san Agustín no se había impuesto un plan estricto ${ }^{32}$ y la de J. Morán cuando sostiene que no es fácil hablar de organicidad ${ }^{33}$. Tampoco podemos ponernos de parte de R. Lorenz que se apoyaba en la deficiente composición del documento monástico para negarle su autoría agustiniana ${ }^{34}$. En efecto, todos los textos que hemos sometido a examen revelan poseer una estructura de fondo uniforme, dentro de lógicas divergencias, explicables por el diverso contexto o momento cronológico. Una estructura en que encaja, sin violencia, la Regla que lleva el nombre del obispo de Hipona. No cabe, pues, sino afirmar que la Regla es más que una retahíla de prescripciones; tiene una estructura definida y, además, sumamente armoniosa. En ella y desde ella hemos podido descubrir a san Agustín como un hombre diestro en arquitectura literaria.

Según G. Lawless la Regla no es en modo alguno un «self-conscious document» como el magnum et arduum opus, el De ciuitate Dei35. Si se refiere a aspectos cuantitativos, nada hay que objetar, pues no admite comparación con ella; si no, hay que mostrar el desacuerdo. La Regla es un documento tan «self-conscious», si no más, como cualquier otro documento agustiniano, si la juzgamos por la estructura detectada. A pesar de no haberla descubierto más que en sus trazos generales, L. Verheijen hablaba de «una pequeña maravilla de construcción» 36 . Y en verdad que lo es. Pero su estructura es más elaborada aún de lo que él juzgaba, aunque más simple de lo que creía A. de Vogüé, quien superponía, sin armonizarlos bien y sin cubrir debidamente la totalidad del documento, tres principios estructurantes ${ }^{37}$; también más compleja de lo que suponía A. Manrique ${ }^{38}$ e incluso Sor Agatha

32. Cf. D. SAnCHIS, Pauvreté monastique et Charité fraternelle chez saint Augustin. Note sur le plan de la Règle, en Augustiniana 8 (1958) 10.

33. «Si è voluto cercare una certa logica nella composizione e nel disporre i diversi cosi detti capituli della Regola. Benchè i titoli dei capitoli e la divisione stessa non sia autentica, stando solo al testo senza aggiunte, non è facile parlare di organicità. Sarebbe d'altra parte presumibilmente fuori del modo spontaneo, benchè pensato, nel quale sembra essere scritta» (L'equilibrio nella Spiritualità della "Regola" di S. Agostino, Augustinianum, Roma 1968, p. 11-12).

34. Cf. Die Anfänge des abendländischen Mönchtum im 4. Jahrhundert, en Zeitschrift für Kirchengeschichte 77 (1966) 1-61; en concreto, p. 60-61.

35. Augustine of Hipo, p. 70.

36. Le De sancta uirginitate, p. 266 NA II, 178.

37. Cf. Estructura (I), en Estudio Agustiniano 32 (1997) 411-412.

38. Cf. Teología agustiniana, pp. 362ss. O también F. Moriones que, de modo similar, distinguía en la Regla un principio básico y luego principios auxiliares (Espiritualidad agustinorecoleta. II. Carácter comunitario y apostólico del carisma agustiniano, Madrid 1989, pp. 225245 y 247-277 respectivamente). O R. Lazcano que considera «todo el resto» de la Regla como una explicitación posterior del principio del «alma sola» (cf. Notas sobre la obediencia en $S$. Agustín, en Revista Agustiniana de Espiritualidad 25 [1985] 219-236: en comcreto, 233-234). O 
Mary39; más orgánica de lo que resulta de la propuesta de M. Neusch40; más armónica que la presentación hecha por Sor Marie-Ancilla ${ }^{41} \mathrm{y}$, por supuesto, más «ordenada» de lo que pensaba L. Rubio, quien, aun en su optimismo, se quedó corto ${ }^{42}$. A la luz de todo lo anterior, no se puede sostener que la Regla sea un simple «torso», «un mero fragmento, un rostro desconchado, extraído de unas excavaciones ${ }^{43}$.

Independientemente de su extensión, una obra así no se escribe a vuela pluma. A su puesta por escrito ha precedido necesariamente un período de reflexión. Estamos ante un documento de estructura elaborada, pero en ningún modo rebuscada. Elaborada con tiempo, no rebuscada en el momento de escribirla con fines estéticos; trazada, se podría decir, con lápiz, no con rotulador de color chillón. La suya es algo más que la estructura pensada para un pequeño documento escrito. De los artículos anteriores se puede deducir que propiamente se debería hablar más bien de una estructura mental del santo que ha encontrado en la Regla su plasmación escrita, una entre otras.

\section{(Continuará)}

PÍO DE LUIS

Estudio Teológico Agustiniano

Valladolid

T. van Bavel para quien «All the other chapters can be seen as elaborations and applications of the inspiring principles effered in the first chapter» ("And honour God in one another» (Rule of Augustine 1,8), en Homo Spiritalis. Festgabe für Luc Verheijen OSA zu seinem 70. Geburtstag, hrg. von C. Mayer unter Mitwirkung von K. H. Chelius, Würzburg 1987, 195-206; en concreto, p. 195.

39. La autora ve la Regla estructurada en dos partes, más o menos iguales en extensión; el criterio que las divide lo señala con estas palabras: "The first part is concerned with the place of individuals in the community -their aspirations, their weaknesses, and their sins; the second part is concerned with the life of the group as a whole. The turning point comes with the first mention of the word «love» (dilectio) [R IV,10]» (The Rule of Saint Augustin. An Essay in Understanding, Augustinian Press, Villanova 1992, p. 13). Ha sido perspicaz para advertir que el concepto «amor», expresado con diferentes términos latinos, sólo aparece en la segunda parte de la Regla y el papel preponderante que en ella tienen las relaciones interpersonales. Pero no es suficiente criterio para estructurar el documento en su totalidad. Por poner sólo un caso, según tal criterio, el capítulo octavo habría que excluirlo de la segunda parte de la Regla. Lo que en él se prescribe mira al individuo en cuanto tal.

40. Cf. La structure de la Règle, p. 14-15.

41. La Règle de saint Augustin, París 1996, p. 61-62.

42. La norma fundamental de la vida monástica según San Agustín y otras reflexiones en torno a la Regula Augustini, en La ciudad de Dios 183 (1970) 189-235. «En la regula Augustini hay un orden, el mejor, tal vez, de los varios que cabían en un documento de esta clase» (p. 191).

43. L. Cilleruelo, Comentario a la Regla de san Agustín, Valladolid 1994, p. 560.559. 